K. Nomizu

Nagoya Math. J.

Vol. 60 (1976), 181-188

\title{
A CHARACTERIZATION OF THE VERONESE VARIETIES*
}

\author{
KATSUMI NOMIZU
}

Let $P^{m}(\boldsymbol{C})$ be the complex projective space of dimension $m$. In a previous paper [2] we have proved

THEOREM A. Let $f$ be a Kaehlerian immersion of a connected, complete Kaehler manifold $M^{n}$ of dimension $n$ into $P^{m}(C)$. If the image $f(\tau)$ of each geodesic $\tau$ in $M^{n}$ lies in a complex projective line $P^{1}(C)$ of $P^{m}(C)$, then $f\left(M^{n}\right)$ is a complex projective subspace of $P^{m}(C)$, and $f$ is totally geodesic.

In the present note, we shall first provide a much simpler geometric proof of this result and then give a characterization of the Veronese varieties by means of the notion of circles in $P^{m}(C)$. Generally, a curve $x(t)$ with arc-length parameter $t$ in a Riemannian manifold is called a circle if there exists a field of unit vectors $Y_{t}$ along the curve, which, together with the unit tangent vectors $X_{t}$, satisfies the differential equations

$$
\nabla_{t} X_{t}=k Y_{t} \text { and } \nabla_{t} Y_{t}=-k X_{t},
$$

where $k$ is a positive constant (see [4]).

By the Veronese variety we mean the imbedding of $P^{n}(C)$ into $P^{m}(C)$, where $m=n(n+3) / 2$, which is defined as follows. Let $S^{2 n+1}$ be the unit sphere in the complex vector space $C^{n+1}$ with the standard hermitian inner product $(z, w)$ and corresponding real inner product $\langle z, w\rangle=\operatorname{Re}(z, w)$. On the other hand, the set of all complex symmetric matrices of degree $n+1$ can be considered as the vector space $C^{m+1}$, where $m=n(n+3) / 2$, in which the standard hermitian inner product can be expressed by

$$
(A, B)=\operatorname{trace} A \bar{B}, \quad A, B \in C^{m+1} .
$$

The mapping $v$ which takes $x \in C^{n+1}$ into $x^{t} x \in C^{m+1}$ maps $S^{2 n+1}$ into the

Received June 10, 1975.

* Work supported by NSF Grant GP-38582X. 
unit sphere $S^{2 m+1}$ of $C^{m+1}$, and induces a holomorphic imbedding of $P^{n}(C)$ into $P^{m}(C)$. If we choose the Fubini-Study metrics of constant holomorphic curvature $c(>0)$ for $P^{m}(C)$ and $c / 2$ for $P^{n}(C)$, then the imbedding is isometric. This is what we call the Veronese imbedding.

We now state our new result.

THEOREM B. Let $f$ be a Kaehlerian immersion of a connected, complete Kaehler manifold $M^{n}$ of dimension $n$ into $P^{m}(C)$ with FubiniStudy metric. The image $f(\tau)$ of each geodesic $\tau$ in $M^{n}$ is a circle in $P^{m}(C)$ if and only if $f$ is congruent (by a holomorphic isometry of $P^{m}(C)$ ) to $i \circ v$, where $v$ is the Veronese imbedding of $P^{n}(C)$ into $P^{m^{\prime}}(C)$, with $m^{\prime}=n(n+3) / 2$, and $i$ is the totally geodesic inclusion of $P^{m^{\prime}}(\boldsymbol{C})$ into $P^{m}(C)$.

\section{Simpler proof of Theorem A.}

Let $x_{0}$ be a point of $M^{n}$ and let $M^{*}$ be the complete totally geodesic complex submanifold (namely, $n$-dimensional projective subspace $P^{n}(C)$ ) through the point $f\left(x_{0}\right)$ and tangent to $f\left(M^{n}\right)$, that is, the tangent space $T_{f\left(x_{0}\right)}\left(M^{*}\right)$ equals $f_{*}\left(T_{x_{0}}\left(M^{n}\right)\right.$ ), where $f_{*}$ denotes the differential of $f$.

Let $\tau$ be an arbitrary geodesic in $M^{n}$ starting at $x_{0}$. By assumption, there is a complex projective line $P^{1}(C)$ which contains $f(\tau)$. If $X$ denotes the initial tangent vector of $\tau$ at $x_{0}$, then $f_{*}(X)$ is tangent to $P^{1}(C)$. If we denote by $J$ the complex structure of $P^{m}(C)$ as well as that of $M^{n}$, then the vector $J f_{*}(X)=f_{*}(J X)$ is tangent to $P^{1}(C)$. It follows that $T_{f\left(x_{0}\right)}\left(P^{1}(C)\right)$ is spanned by $f_{*}(X)$ and $f_{*}(J X)$. On the other hand, these two vectors are contained in $f_{*}\left(T_{x_{0}}\left(M^{n}\right)\right)=T_{f\left(x_{0}\right)}\left(M^{*}\right)$. Thus $T_{f\left(x_{0}\right)}\left(P^{1}(C)\right)$ $\subset T_{f\left(x_{0}\right)}\left(M^{*}\right)$. Since $P^{1}(C)$ and $M^{*}$ are totally geodesic in $P^{m}(C)$, it follows that $P^{1}(C)$ is contained in $M^{*}$; thus $f(\tau)$ is contained in $M^{*}$. Since $\tau$ is an arbitrary geodesic in $M$, we have $f(M)=M^{*}$.

\section{Veronese imbedding.}

We shall show that the Veronese imbedding $v$ of $P^{n}(C)$ into $P^{m}(C)$ with $m=n(n+3) / 2$ has the property that the image of each geodesic in $P^{n}(C)$ is a circle in $P^{m}(C)$. This property does not depend on the choice of a positive constant $c$ which we choose for the holomorphic sectional curvature of $P^{m}(C)$ (and that of $P^{n}(C)$ will be $c / 2$ ). We recall how geometry of $P^{m}(C)$ is related to that of $S^{2 m+1}$. The standard fibration $\pi: S^{2 m+1} \rightarrow P^{m}(C)$ is a principal $S^{1}$-bundle. It has a connection whose 
horizontal subspaces $Q_{x}, x \in S^{2 m+1}$, are given by

$$
Q_{x}=\left\{X \in C^{m+1} ;\langle X, x\rangle=\langle X, i x\rangle=0\right\} .
$$

The projection $\pi_{*}$ maps $Q_{x}$ isomorphically onto the tangent space $T_{u}\left(P^{m}(C)\right)$, where $u=\pi(x)$. If we let

$$
g\left(\pi_{*} X, \pi_{*} Y\right)=(4 / c)\langle X, Y\rangle, \quad X, Y \in Q_{x},
$$

then $g$ is the Fubini-Study metric with holomorphic sectional curvature $c$ for $P^{m}(C)$. We shall choose $c=4$ (to simplify constant factors in the computations that follow). Let us denote by $\nabla^{\prime}$ the Riemannian connection for $S^{2 m+1}$ and by $\tilde{\nabla}$ the Kaehlerian connection for $P^{m}(C)$. We formulate the relationship between $\nabla^{\prime}$ and $\tilde{\nabla}$ (see [3], Proposition 3) in the following form. A curve in $S^{2 m+1}$ is said to be horizontal if its tangent vectors are horizontal.

LEMMA 1. Let $x_{t}$ be a horizontal curve in $S^{2 m+1}$ and $u_{t}=\pi\left(x_{t}\right)$. If $Z_{t}$ is a horizontal vector field along $x_{t}$ and if $W_{t}=\pi_{*}\left(Z_{t}\right)$, then $\tilde{\nabla}_{t} W_{t}=$ $\pi_{*}\left(\nabla_{t}^{\prime} Z_{t}\right)$.

LEMMA 2. If $x_{t}$ is a horizontal curve in $S^{2 m+1}$ with arc-length parameter $t$, then $\nabla_{t}^{\prime} X_{t}$, where $X_{t}$ denotes the tangent vector, is horizontal.

Proof. We have

$$
\nabla_{t}^{\prime} X_{t}=d X / d t+x_{t}
$$

Since $x_{t}$ is horizontal, we have $\left\langle X_{t}, i x_{t}\right\rangle=0$ and hence

$$
\left\langle d X / d t, i x_{t}\right\rangle+\left\langle X_{t}, i X_{t}\right\rangle=0 \text {. }
$$

But $\left\langle X_{t}, i X_{t}\right\rangle=0$ so that $\left\langle d X / d t, i x_{t}\right\rangle=0$. Thus we obtain

$$
\left\langle\nabla_{t}^{\prime} X_{t}, i x_{t}\right\rangle=\left\langle d X / d t, i x_{t}\right\rangle+\left\langle x_{t}, i x_{t}\right\rangle=0 .
$$

LEMMA 3. If $x_{t}$ is a circle in $S^{2 m+1}$ which is furthermore a horizontal curve, then $u_{t}=\pi\left(x_{t}\right)$ is a circle in $P^{m}(C)$.

Proof. We have a field of unit vectors $Y_{t}$ along $x_{t}$ such that

$$
\nabla_{t}^{\prime} X_{t}=k Y_{t} \text { and } \nabla_{t}^{\prime} Y_{t}=-k X_{t},
$$

where $k$ is a positive constant and $X_{t}$ is the tangent vector. By Lemma 2, $\nabla_{t}^{\prime} X_{t}$ and hence $Y_{t}$ are horizontal. The tangent vector of $u_{t}$ is given by $U_{t}=\pi_{*}\left(X_{t}\right)$. Consider the field of unit normal vectors $V_{t}=\pi_{*}\left(Y_{t}\right)$; 
note that $\pi_{*}$ is isometric from $Q_{x}$ to $T_{\pi(x)}\left(P^{m}(C)\right)$. By Lemma 1, we have

$$
\tilde{\nabla}_{t} U_{t}=\pi_{*}\left(\nabla_{t}^{\prime} X_{t}\right)=\pi_{*}\left(k Y_{t}\right)=k V_{t}
$$

and, similarly,

$$
\tilde{\nabla}_{t} V_{t}=\pi_{*}\left(\nabla_{t}^{\prime} Y_{t}\right)=\pi_{*}\left(-k X_{t}\right)=-k U_{t} .
$$

Thus $u_{t}$ is a circle in $P^{m}(C)$.

Now we shall prove our assertion about the Veronese imbedding. We observe that the unitary group $U(n+1)$ acts naturally on $S^{2 n+1}$ and $P^{n}(C)$ as a group of isometries. Each geodesic $\tau$ in $P^{n}(C)$ is congruent by a transformation belonging to $U(n+1)$ to the curve with homogeneous coordinates $(\cos t, \sin t, 0, \cdots, 0)$. On the other hand, we can let $U(n+1)$ act on the space $C^{m+1}$ of all complex symmetric matrices of degree $n+1$ by $Z \rightarrow A Z^{t} A$, where $Z \in C^{m+1}$ and $A \in U(n+1)$. This action preserves inner product in $C^{m+1}$ and thus induces the action of $U(n+1)$ on $S^{2 m+1}$ and $P^{m}(C)$ as a group of isometries. Now the Veronese imbedding $v$ is equivariant relative to the actions of $U(n+1)$ on $P^{n}(C)$ and on $P^{m}(C)$.

It is thus sufficient to prove the following. Let $\tau$ be the geodesic $w_{t}$ in $P^{n}(C)$ given by $w_{t}=\pi\left(z_{t}\right)$, where $z_{t}=(\cos (t / \sqrt{2}), \sin (t / \sqrt{2}), 0$, $\cdots, 0)$ is a curve on $S^{2 n+1}$. Since the holomorphic sectional curvature of $P^{n}(C)$ has been chosen to be 2 , we have

$$
\|d w / d t\|^{2}=2\|d z / d t\|^{2}=1,
$$

which shows that $t$ is the arc-length parameter for the geodesic $w_{t}$. Let

$$
x_{t}=v\left(z_{t}\right), \quad u_{t}=v\left(w_{t}\right) \text { so that } u_{t}=\pi\left(x_{t}\right) .
$$

We wish to show that $u_{t}$ is a circle in $P^{m}(C)$. The curve $x_{t}$ on $S^{2 m+1}$ can be represented simply by the first $2 \times 2$ block of the form

$$
\left[\begin{array}{cc}
\cos ^{2}(t / \sqrt{2}) & \sin (t / \sqrt{2}) \cos (t / \sqrt{2}) \\
\sin (t / \sqrt{2}) \cos (t / \sqrt{2}) & \sin ^{2}(t / \sqrt{2})
\end{array}\right]
$$

since the other components are all 0 . The tangent vectors $X_{t}$ of the curve $x_{t}$ are represented in the same sense by

$$
X_{t}=(1 / \sqrt{2})\left[\begin{array}{rr}
-\sin (\sqrt{2} t) & \cos (\sqrt{2} t) \\
\cos (\sqrt{2} t) & \sin (\sqrt{2} t)
\end{array}\right]
$$


Since $\left\langle X_{t}, i x_{t}\right\rangle=0, x_{t}$ is a horizontal curve in $S^{2 m+1}$. If we show that it is a circle in $S^{2 m+1}$, then Lemma 3 implies that $u_{t}=\pi\left(x_{t}\right)$ is a circle in $P^{m}(C)$.

We have

$$
d X / d t=\left[\begin{array}{rr}
-\cos (\sqrt{2} t) & -\sin (\sqrt{2} t) \\
-\sin (\sqrt{2} t) & \cos (\sqrt{2} t)
\end{array}\right]
$$

The vector

$$
\nabla_{t}^{\prime} X_{t}=d X / d t+x_{t}
$$

is also horizontal (since its components are real) and has length 1, because

$$
\begin{aligned}
& \left\langle d X / d t+x_{t}, d X / d t+x_{t}\right\rangle \\
& \quad=\langle d X / d t, d X / d t\rangle+2\left\langle x_{t}, d X / d t\right\rangle+\left\langle x_{t}, x_{t}\right\rangle \\
& \quad=2+2(-1)+1=1,
\end{aligned}
$$

by virtue of $\left\langle x_{t}, d X / d t\right\rangle=-\left\langle d x / d t, X_{t}\right\rangle=-1$.

We thus set $Y_{t}=d X / d t+x_{t}$, namely, $\nabla_{t}^{\prime} X_{t}=Y_{t}$. Since $\left\langle Y_{t}, X_{t}\right\rangle=0$, we have

$$
\begin{aligned}
\nabla_{t}^{\prime} Y_{t}= & d Y / d t=d^{2} X / d t^{2}+X_{t} \\
= & \sqrt{2}\left[\begin{array}{rr}
\sin (\sqrt{2} t) & -\cos (\sqrt{2} t) \\
-\cos (\sqrt{2} t) & -\sin (\sqrt{2} t)
\end{array}\right] \\
& +(1 / \sqrt{2})\left[\begin{array}{rr}
-\sin (\sqrt{2} t) & \cos (\sqrt{2} t) \\
\cos (\sqrt{2} t) & \sin (\sqrt{2} t)
\end{array}\right] \\
= & (1 / \sqrt{2})\left[\begin{array}{rr}
\sin (\sqrt{2} t) & -\cos (\sqrt{2} t) \\
-\cos (\sqrt{2} t) & -\sin (\sqrt{2} t)
\end{array}\right]=-X_{t} .
\end{aligned}
$$

Thus we have shown that $x_{t}$ is a circle of curvature $k=1$.

\section{Proof of Theorem B.}

We now finish the proof of Theorem B. Let $f$ be a Kaehlerian immersion of a complete Kaehler manifold $M^{n}$ into $P^{m}(C)$ with the property that for each geodesic $\tau$ in $M^{n}$ the image $f(\tau)$ is a circle in $P^{m}(C)$. We shall first show that

(i) the second fundamental form $\alpha$ is parallel;

(ii) $f$ is isotropic, that is, $\|\alpha(X, X)\|$ is equal to a constant for all unit tangent vectors $X$ to $M^{n}$ at each point; 
(iii) $M^{n}$ has constant holomorphic curvature.

Let $x_{t}$ be a geodesic on $M^{n}$ with tangent vectors $X_{t}$ of length 1 . Denoting by $\tilde{\nabla}$ and $\nabla$ the Kaehlerian connections of $P^{m}(C)$ and $M^{n}$, respectively, we have

$$
\tilde{\nabla}_{t} X_{t}=\nabla_{t} X_{t}+\alpha\left(X_{t}, X_{t}\right)=\alpha\left(X_{t}, X_{t}\right),
$$

where $\alpha$ is the second fundamental form. We obtain

$$
\tilde{\nabla}_{t}^{2} X_{t}=-A_{\alpha\left(X_{t}, X_{t}\right)} X_{t}+\nabla_{t}^{\frac{1}{\alpha}} \alpha\left(X_{t}, X_{t}\right),
$$

where $A$ is the second fundamental tensor and $\nabla^{\perp}$ the normal connection. On the other hand, since $f\left(x_{t}\right)$ is a circle by assumption, there exists a field of unit tangent vectors $Y_{t}$ along $x_{t}$ and $k>0$ such that

$$
\tilde{\nabla}_{t} X_{t}=k Y_{t} \text { and } \tilde{\nabla}_{t} Y_{t}=-k X_{t},
$$

thus

$$
\tilde{\nabla}_{t}^{2} X_{t}=-k^{2} X_{t}
$$

From (1) and (2) we obtain

$$
A_{\alpha\left(X_{t}, X_{t}\right)} X_{t}=k^{2} X_{t}
$$

and

$$
\nabla_{t}^{\frac{1}{\alpha}} \alpha\left(X_{t}, X_{t}\right)=0 \text {. }
$$

Since $x_{t}$ is a geodesic in $M^{n}$, the covariant derivative

$$
\left(\nabla_{t}^{*} \alpha\right)\left(X_{t}, X_{t}\right)=\nabla_{t}^{\perp} \alpha\left(X_{t}, X_{t}\right)-\alpha\left(\nabla_{t} X_{t}, X_{t}\right)-\alpha\left(X_{t}, \nabla_{t} X_{t}\right)
$$

is equal to 0 by virtue of (4). Evaluating this at $t=0$ and observing that $X_{0}$ can be an arbitrary unit tangent vector at an arbitrary point of $M^{n}$, we have

$$
\left(\nabla_{X}^{*} \alpha\right)(X, X)=0 \quad \text { for all tangent vectors } X \text { to } M^{n} \text {. }
$$

Since $\left(\nabla_{X}^{*} \alpha\right)(Y, Z)$ is symmetric in $X, Y$ and $Z$, we conclude that $\nabla^{*} \alpha=0$, that is, $\alpha$ is parallel.

From (3) it follows that for any unit tangent vector $X$ to $M^{n}$ there exists a certain constant $k>0$ such that

$$
A_{\alpha(X, X)} X=k^{2} X
$$

If $Y$ is a tangent vector perpendicular to $X$, then 


$$
\left\langle A_{\alpha(X, X)} X, Y\right\rangle=0
$$

so that

$$
\langle\alpha(X, X), \alpha(X, Y)\rangle=0 \quad \text { whenever }\langle X, Y\rangle=0 .
$$

This condition implies that $f$ is isotropic, that is, $\|\alpha(X, X)\|$ is equal to a constant for all unit tangent vectors $X$ at each point (see [6], Lemma 1). It also follows that $M^{n}$ has constant holomorphic sectional curvature (see [6], Lemma 6).

We now wish to prove that $f$ is essentially the Veronese imbedding. Since $\alpha$ is parallel, the first normal spaces (spanned by the range of $\alpha$ at each point) are obviously parallel relative to the normal connection. The (complex) dimension of the normal spaces, say, $p$, is at most $n(n+1) / 2$. It is known [1], Proposition 9, that there is a totally geodesic $P^{n+p}(C)$ in $P^{m}(C)$ such that $f\left(M^{n}\right) \subset P^{n+p}(C)$. We shall see that this immersion $f_{0}$ of $M^{n}$ into $P^{n+p}(C)$ is the Veronese imbedding (and indeed $p=$ $n(n+1) / 2)$.

If $p<n(n+1) / 2$, Theorem 2 of [6] says that $f_{0}$ is totally geodesic. This will mean that the image of a geodesic in $M^{n}$ is a geodesic in $P^{n+p}(C)$ and hence a geodesic in $P^{m}(C)$, contrary to the assumption that it is a circle in $P^{m}(C)$. Hence we must have $p=n(n+1) / 2$. We already know that $M^{n}$ has constant holomorphic sectional curvature. Since the second fundamental form is parallel, it follows from [5], Theorem 4.4, that this constant is half the constant holomorphic sectional curvature of $P^{n+p}(C)$. Moreover, such an immersion $f_{0}$ is rigid. Thus $M^{n}$ is $P^{n}(C)$ with holomorphic sectional curvature, say, 2, if we assume that $P^{m}(C)$ and hence $P^{n+p}(C)$ has holomorphic sectional curvature 4. Now the Veronese imbedding $v$ is a Kaehlerian imbedding of $P^{n}(C)$ into $P^{n+p}(C)$. By rigidity, $f_{0}$ is congruent to $v$ by a holomorphic isometry of $P^{n+p}(C)$. Since this holomorphic isometry can be extended to a holomorphic isometry of $P^{m}(C)$, we can now conclude that $f: M^{n} \rightarrow P^{m}(C)$ is in fact congruent to $i \circ v$, where $v$ is the Veronese imbedding of $P^{n}(C)$ into $P^{n+p}(C), p=n(n+1) / 2$, and $i$ is a totally geodesic inclusion of $P^{n+p}(C)$ into $P^{m}(C)$. We have thus completed the proof of Theorem B.

\section{REFERENCES}

[1] Cecil, T. E.: Geometric applications of critical point theory to submanifolds of complex projective space, Nagoya Math. J., 55 (1974), 5-31. 
[2] Nomizu, K.: Eine Kennzeichnung der projektiven Unterräume in dem komplexen projektiven Raum nach ihren Geodätischen, Math. Z., 137 (1974), 147-150.

[ 3 ] Nomizu K. and Smyth, B.: Differential geometry of complex hypersurfaces II, J. Math. Soc. Japan, 20 (1968), 498-521.

[4] Nomizu, K. and Yano, K.: On circles and spheres in Riemannian geometry, Math. Ann., 210 (1974), 163-170.

[5] Ogiue, K.: Differential Geometry of Kaehler Submanifolds, Advances in Mathematics, vol. 13, no. 1 (1974), 73-114.

[6 ] O'Neill, B.: Isotropic and Kähler immersions, Canadian J. Math., 17 (1965), 907-915.

Brown University 